\title{
Enabling ESSENCE to Process and Export Meaningful Use Syndromic Surveillance Data
}

\author{
Miles Stewart ${ }^{1}$, Wayne Loschen*1 and Taha Kass-Hout ${ }^{2}$ \\ 1Johns Hopkins University Applied Physics Laboratory, Laurel, MD, USA; 2 Public Health Surveillance and Informatics Program Office, \\ Office of Surveillance, Epidemiology, \& Laboratory Services, Centers for Disease Control and Prevention, Atlanta, GA, USA
}

\section{Objective}

The objective of this project is to enable the ESSENCE system to read in, utilize, and export out meaningful use syndromic surveillance data using the Health Level 7 (HL7) v2.5 standard. This presentation will detail the technical hurdles with reading a meaningful use syndromic surveillance data feed containing multiple sources, deriving a common meaning from the varying uses of the standard and writing data out to a meaningful use HL7 2.5 format that can be exported to other tools, such as BioSense 2.0 (2). The presentation will also describe the technologies employed for facilitating this, such as Mirth, and will discuss how other systems could utilize these tools to also support processing meaningful use syndromic surveillance data.

\section{Introduction}

In order to utilize the new meaningful use syndromic surveillance data sets (3) that many public health departments are now receiving, modifications to their systems must be made. Typically this involves enabling the storage and processing of the extra fields the new standard contains. Open source software exists, such as Mirth Connect, to help with reading and interpreting the standard. However, issues with reliably reading data from one source to another arise when the standard itself is misunderstood. Systems that process this data must understand that while the data they receive is in the HL7 v2.5 standard format, the meaning of the data fields might be different from provider to provider. Additional work is necessary to sift through the similar yet disjoint fields to achieve a consistent meaning.

\section{Methods}

This project utilized 3 separate instances of ESSENCE and BioSense 2.0. For both importing and exporting HL7 v2.x standard files, the project used the open source tool Mirth Connect. For importing data the project adapted versions of Tarrant County and Cook County ESSENCE systems in the Amazon GovCloud to receive meaningful use syndromic surveillance data files sent from BioSense 2.0. For exporting data to BioSense 2.0, the project used Mirth Connect to poll the local version of Cook County's ESSENCE database and export the data into an HL7 v2.5 file. The resultant file was sent over secure file transfer protocol (SFTP) to BioSense 2.0. The team then evaluated the process by comparing the data in the local instances of ESSENCE and the corresponding instances hosted on the Internet cloud.

\section{Results}

Many issues were encountered during the reading of the HL7. While the standard suggests that hospitals and hospital systems would all send data in the same fields for the same data, the reality was far different. Although HL7 v2.5 is a standard and there is a defined use for each field, it can be interpreted in many ways. A large portion of time was spent communicating with the local health department to determine exactly what each field meant for a particular hospital. Comparing the Internet cloud and local versions did have some difficulties due to local filtration rules that eliminated non-ER related records from the local Tarrant County system. The project was able to utilize new query features in ESSENCE to filter down to only ER related records on the Internet cloud version to support the comparisons. The project was able to re-use much of the configuration that was created when moving from one jurisdiction to the other. This will help when describing how others may use the same technology in their own systems.

\section{Conclusions}

Reading and interpreting the data consistently from a data feed containing multiple sources can be challenging. Confusion with the HL7 v2.3 or 2.5 standards causes many health organizations to transmit data in inconsistent ways that betrays the notion of a messaging standard. However, with the tools this project have created and the lessons we have learned, the pain of implementing meaningful use syndromic surveillance data into a system can be reduced.

\section{Keywords}

Analytics; Electronic Medical Records for Public Health; Interoperability; Meaningful Use; Internet Cloud

\section{Acknowledgments}

The ESSENCE in the Cloud initiative is supported by the CDC's Division of Notifiable Diseases and Healthcare Information (DNDHI) BioSense Program.

\section{References}

1) Kass-Hout, et al, CDC's BioSense 2.0: Bringing Together the Science and Practice of Public Health Surveillance, AJPM Prevention In Practice, November 15, 2011

2) PHIN Messaging Guide for Syndromic Surveillance: Emergency Department and Urgent Care Data. Accessed August 30, 2012: http://www.cdc.gov/ehrmeaningfuluse/Syndromic.html.

\section{*Wayne Loschen}

E-mail: wayne.loschen@jhuapl.edu 